\title{
THE DISTURBANCE OF A MILLISECOND PULSAR MAGNETOSPHERE
}

\author{
R. M. Shannon ${ }^{1,2}$, L. T. Lentati ${ }^{3}$, M. Kerr ${ }^{1}$, M. Bailes ${ }^{4}$, N. D. R. Bhat ${ }^{2}$, W. A. Coles ${ }^{5}$, S. Dai $^{1}$, J. Dempsey $^{6}$, G. Hobbs $^{1}$, \\ M. J. Keith ${ }^{7}$, P. D. Lasky ${ }^{8}$, Y. Levin ${ }^{8}$, R. N. Manchester ${ }^{1}$, S. OsŁowski ${ }^{4}$, V. Ravi ${ }^{9}$, D. J. Reardon ${ }^{1,8}$, P. A. Rosado ${ }^{4}$, \\ R. SPIEWAK ${ }^{10}$, W. van Straten ${ }^{4}$, L. Toomey ${ }^{1}$, J.-B. WAng ${ }^{11}$, L. Wen ${ }^{12}$, X.-P. You ${ }^{13}$, and X.-J. Zhu ${ }^{12}$ \\ ${ }^{1}$ CSIRO Astronomy and Space Science, Australia Telescope National Facility, Box 76, Epping, NSW 1710, Australia; ryan.shannon@csiro.au \\ ${ }^{2}$ International Centre for Radio Astronomy Research, Curtin University, Bentley, WA 6102, Australia \\ ${ }^{3}$ Astrophysics Group, Cavendish Laboratory, JJ Thomson Avenue, Cambridge CB3 0HE, UK \\ ${ }^{4}$ Centre for Astrophysics and Supercomputing, Swinburne University of Technology, P.O. Box 218, Hawthorn, VIC 3122, Australia \\ 5 Department of Electrical and Computer Engineering, University of California, San Diego, La Jolla, CA 92093, USA \\ ${ }^{6}$ CSIRO Information Management \& Technology, Box 225, Dickson, ACT 2602, Australia \\ ${ }^{7}$ Jodrell Bank Centre for Astrophysics, University of Manchester, M13 9PL, UK \\ ${ }^{8}$ Monash Centre for Astrophysics, School of Physics and Astronomy, Monash University, VIC 3800, Australia \\ ${ }^{9}$ Cahill Center for Astronomy and Astrophysics, California Institute of Technology, MC 249-17, Pasadena, CA 91125, USA \\ ${ }^{10}$ Department of Physics, University of Wisconsin-Milwaukee, Box 413, Milwaukee, WI 53201, USA \\ ${ }^{11}$ Xinjiang Astronomical Observatory, Chinese Academy of Sciences, 150 Science 1-Street, Urumqi, Xinjiang 830011, China \\ ${ }^{12}$ School of Physics, University of Western Australia, Crawley, WA 6009, Australia \\ ${ }^{13}$ School of Physical Science and Technology, Southwest University, Chongqing 400715, China \\ Received 2016 July 5; revised 2016 August 4; accepted 2016 August 5; published 2016 August 22
}

\begin{abstract}
Pulsar timing has enabled some of the strongest tests of fundamental physics. Central to the technique is the assumption that the detected radio pulses can be used to accurately measure the rotation of the pulsar. Here, we report on a broadband variation in the pulse profile of the millisecond pulsar J1643-1224. A new component of emission suddenly appears in the pulse profile, decays over four months, and results in a permanently modified pulse shape. Profile variations such as these may be the origin of timing noise observed in other millisecond pulsars. The sensitivity of pulsar-timing observations to gravitational radiation can be increased by accounting for this variability.
\end{abstract}

Key words: pulsars: general - pulsars: individual (PSR J1643-1224) - stars: neutron

\section{INTRODUCTION}

The measurement of arrival times (TOAs) of pulses from radio pulsars has enabled physical tests impossible in terrestrial laboratories or elsewhere in the universe, including studies of nuclear equations of state (Demorest et al. 2010) and strongfield tests of the general theory of relativity (Kramer et al. 2006). Another goal of precision timing is the direct detection of gravitational radiation. By monitoring TOAs from an array of spin-stable millisecond pulsars (MSPs) for months to decades, it is likely possible to detect the distortions in TOAs induced by gravitational waves traveling through the solar neighborhood and to distinguish their quadropolar signature from contaminating noise sources (Foster \& Backer 1990; Hobbs et al. 2010; Tiburzi et al. 2016). Two properties of pulsars are central to the power of the pulsar-timing technique. First, pulsars are rotationally stable, with the spin of the pulsar well modeled deterministically and every rotation of the pulsar accounted for over year- to decades-length observing spans. Second is the assumption is that the pulsar radio emissionlocated in the diffuse pulsar magnetosphere tens to thousands of kilometers above the neutron-star surface-is anchored to the star.

Although individual pulses show markedly different morphology (e.g., Cordes \& Shannon 2010), a third assumption is that the longitude-resolved average emission profile converges toward a stable shape after many rotations of the pulsar. It has been known for decades that many slowly spinning pulsars have profiles that switch between distinct emission modes on timescales of minutes to hours (referred to as mode-switching). More recently, Lyne et al. (2010) identified subtle pulse-profile variations in long-term observations of young pulsars, with the pulsars switching modes on month to decade timescales, and the state switches correlated with discrete changes in spindown.

Pulse-shape variations can also be caused by external factors. For example, precession of the pulsar spin axis has been observed for pulsars in the most compact, relativistic orbits (Weisberg et al. 1989). As the orientation of the axis changes with respect to our line of sight, regions of different radio luminosity are beamed toward the Earth. Interstellar propagation effects are also predicted to cause pulse-profile variations (Cordes \& Shannon 2010). As the pulsar and Earth move, the line of sight samples a different column of interstellar material, which can potentially cause variable pulse broadening.

There is only modest evidence for pulse-shape variations in MSPs central to pulsar timing array observations. Claims of epoch-to-epoch mode-switching in the millisecond pulsar J1022+1001 (Kramer et al. 1999) have been disputed. These profile variations can instead be attributed to instrumentalcalibration errors (Hotan et al. 2004a; van Straten 2013) and spectral evolution of the profile (Ramachandran \& Kramer 2003; Dai et al. 2015; Liu et al. 2015). Other well-monitored MSPs have shown no evidence for pulse-profile evolution over decades-long timescales (Shao et al. 2013).

\section{OBSERVATIONS}

As part of the Parkes Pulsar Timing Array project (PPTA; Manchester et al. 2013), we have been observing PSR J1643 -1224 with the $64 \mathrm{~m}$ Parkes telescope at a $\approx 3$ week cadence since 2003. At each epoch, the pulsar is normally observed in three frequency bands: at a frequency close to $1400 \mathrm{MHz}$ using either the central feed of the $20 \mathrm{~cm}$ multibeam system or the $\mathrm{H}$ $-\mathrm{OH}$ receiver, and simultaneously at frequencies close to 

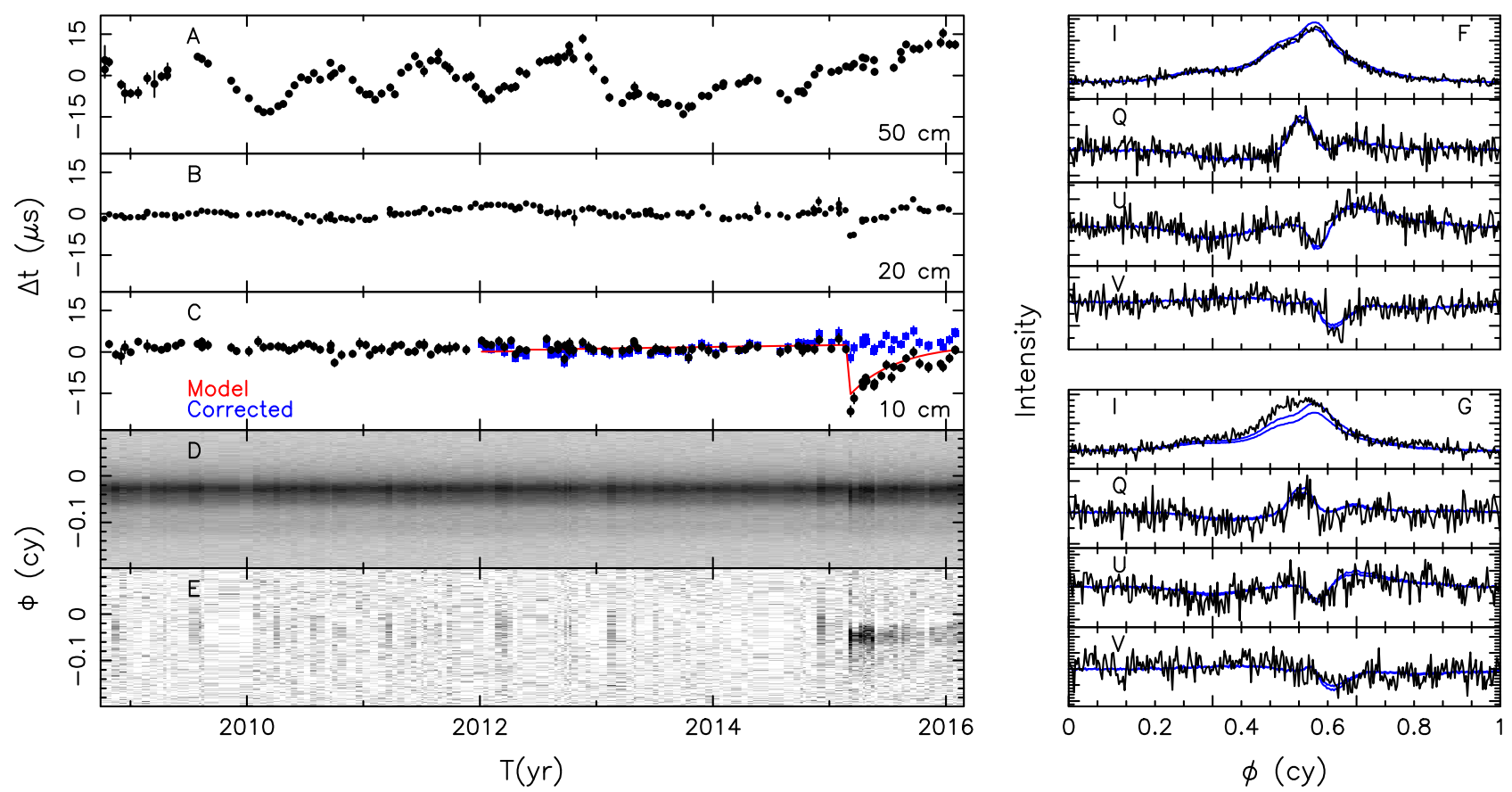

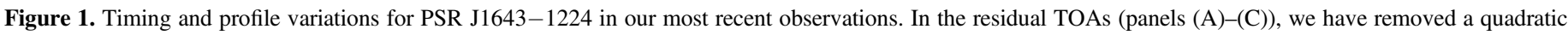

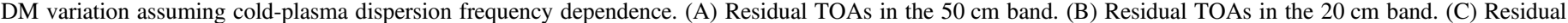

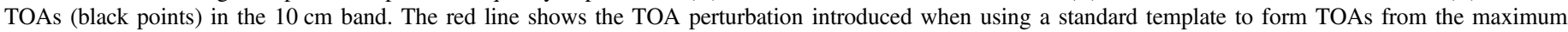

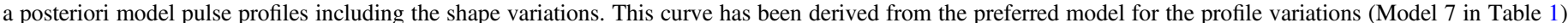

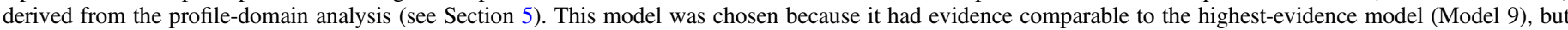

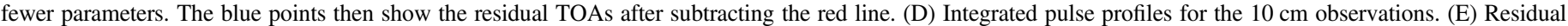

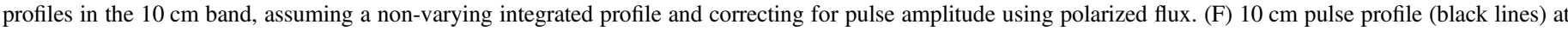

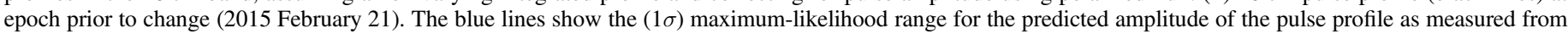
the polarized flux. (G) $10 \mathrm{~cm}$ pulse profile at epoch after change (2015 March 7).

$3100 \mathrm{MHz}$ and $700 \mathrm{MHz}$ using the dual-band $10 \mathrm{~cm} / 50 \mathrm{~cm}$ system. Observations have been made with a range of backends, but the observations central to the results here were made with the mark-3 and -4 Parkes digital filterbank systems (PDFB3/4) and the CASPER-Parkes-Swinburne recorder (CASPSR). Since 2014 May, the PDFB3 backend has been unavailable because of hardware failure. Since 2015 June, the $50 \mathrm{~cm}$ band has been affected by radio-frequency interference from a $4 \mathrm{G}$ base station $10 \mathrm{~km}$ north of the telescope in nearby Alectown

The backend systems use fundamentally different processing techniques to process the voltage time series data: the PDFB systems employ digital polyphase filterbanks produced on field-programmable gate arrays and CASPSR employs coherent dedispersion on graphics-processing units. Both systems use online folding to produce average spectra (in four pseudoStokes parameters) that sample pulse phase and average over time.

The observations were calibrated and analyzed using standard pulsar processing techniques as implemented in the code PSRCHIVE (Hotan et al. 2004b) within the PPTA datareduction pipeline (Manchester et al. 2013). In brief, median filters were applied to the spectra to mitigate the effects of radio-frequency interference and variable differential gain and phase of the system were measured using regular observations of a noise-diode signal, injected $45^{\circ}$ from the linear feeds. The flux-density scale of the observations was determined by relating the strength of the noise-diode signal to regular observations of the radio galaxy Hydra A. For observations made with the $20 \mathrm{~cm}$ multibeam system, we also corrected for cross-coupling in the feed.

\section{TIMING ANALYSIS}

Evidence for changes in pulse shape were first identified in timing analyses. The TOAs were produced by cross-correlating the calibrated Stokes-I profiles with an analytic template, constructed from the average of observations from 2008 to 2014 (Dai et al. 2015). Analysis of the TOAs was conducted using the TEMPO2 (Edwards et al. 2006) and TEMPONEST (Lentati et al. 2014) codes. TOAs were referred to the solar system barycenter using the DE421 ephemeris published by the Jet Propulsion Laboratory and the 2015 realization of terrestrial time published by the International Bureau of Weights and Measures.

In Figure 1, we show the maximum-likelihood residual TOAs obtained for PSR J1643-1224, individually in the $50 \mathrm{~cm}$ (panel (A)), $20 \mathrm{~cm}$ (panel (B)), and $10 \mathrm{~cm}$ bands (panel (C)). The variations in interstellar dispersion were modeled with a quadratic (in time) polynomial, using only $10 \mathrm{~cm}$ and $20 \mathrm{~cm}$ observations. This only removes the lowest fluctuation frequency dispersion-measure variations, but is sufficient for our analysis.

Relative to this model, we find that the $50 \mathrm{~cm}$ observations show annual TOA variations inconsistent with the $10 \mathrm{~cm}$ and $20 \mathrm{~cm}$ observations. The level of signal in these variations scale $\propto \lambda^{4}$, suggesting that they are associated with scattering in the interstellar medium (Lentati et al. 2016). They are likely the result of the Earth-pulsar line of sight resampling the same 


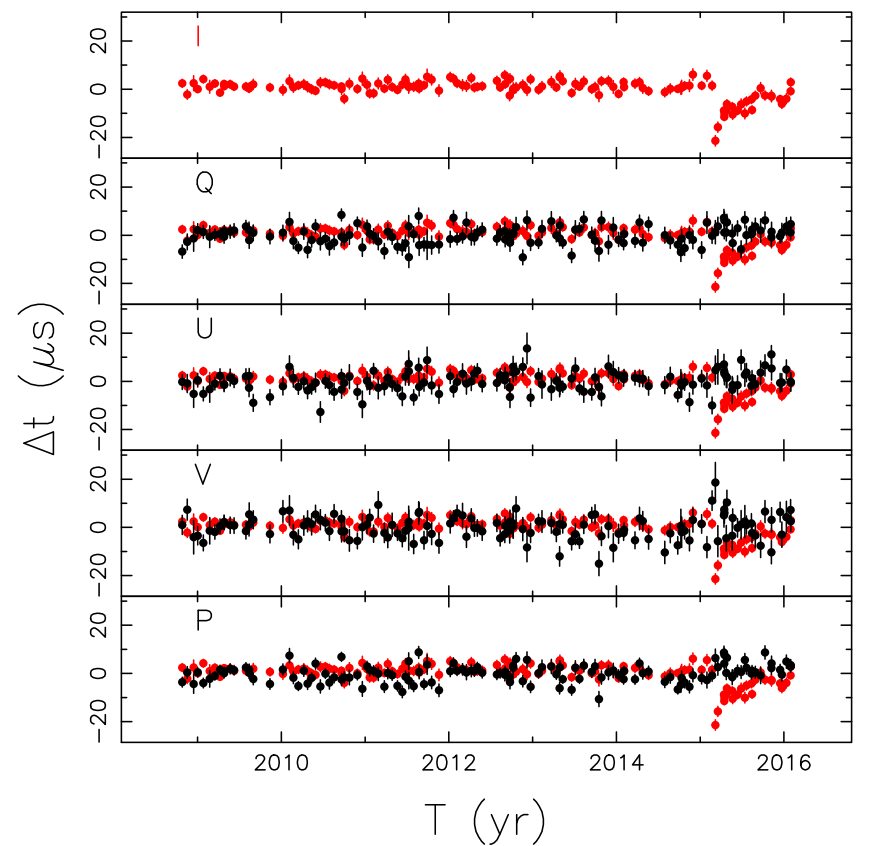

Figure 2. Maximum-likelihood residual TOAs for PSR J1643-1224, timing in Stokes parameters $I, Q, U$, and $V$ as listed on the plot. The bottom panel (labeled $P$ ) shows the average residuals of the polarized components, weighted by their uncertainties. The Stokes-I TOAs are plotted in red in all panels.

region of the ISM for this low-velocity pulsar $\left(v_{\perp} \approx 25 \mathrm{~km} \mathrm{~s}^{-1}\right.$; Reardon et al. 2016).

The largest departure of the residuals occurred since 2015 March, predominantly in the shortest-wavelength $(10 \mathrm{~cm})$ band. Between observations of 2015 February 21 and March 7 the apparent TOAs in this band arrived $\approx 25 \mu$ s earlier. In the $20 \mathrm{~cm}$ band, the TOAs shifted by $\approx 10 \mu$ s between 2015 February 19 and March 6. In contrast, no significant arrival-time variations were observed at $50 \mathrm{~cm}$, meaning that these variations are unlikely to be caused by changes in the ISM. Since then, the offset has decreased in amplitude, with the pulses now arriving $\approx 5 \mu$ s earlier than the long-term pre-2015 level.

These variations are unlikely to be instrumental in origin. In the $20 \mathrm{~cm}$ band, the TOA variations are common to two backends (PDFB4 and CASPSR). In the $10 \mathrm{~cm}$ band, other pulsars observed with the same backend (PDFB4) in the same firmware configuration do not show this offset, with a limit on the change of arrival time of $<100 \mathrm{~ns}$ for the most stable pulsar in the sample, PSR J1909-3744 (Shannon et al. 2015). The lack of similar variations in other pulsars excludes other telescope-dependent (but backend-independent) origins, including an incorrect time or frequency reference at the observatory. In particular, PSR J1643-1224 is less susceptible to polarization calibration errors than many other pulsars in the PPTA sample (van Straten 2013).

The TOAs in other Stokes components do not show the same sudden variation or recovery. In Figure 2, we show the TOAs determined from timing individual Stokes parameters. None showed the magnitude of variation observed in Stokes I. In contrast, bona fide arrival-time variations would affect all Stokes components equally. A pulse-shape change could manifest differently in the four Stokes components and would also introduce observed apparent arrival-time variation.

\section{PULSE-SHAPE VARIATIONS}

Identifying and characterizing changes in pulse shape are complicated by the unknown a priori pulse arrival times and flux densities. Pulse flux densities show marked variations from epoch to epoch as the received strength is modulated by diffractive and refractive scintillation of the pulsar radiation in the interstellar medium (Rickett 1990). The pulse arrival time can be predicted from a model, but will be incorrect if an unmodeled or poorly modeled process is present in the observations. When pulse profiles are shifted and scaled for these unknown parameters, much of the shape variations can be absorbed into these two parameters.

Because the polarized emission of PSR J1643-1224 is nearly unaffected by the shape change, it can be used to measure the expected amplitude and phase of the total intensity pulse profile. Prior to the 2015 March event, we assumed that the pulse shape was constant and then extrapolated the longterm model after the event.

An amplitude estimator for Stokes $I$ was calculated by averaging the individual amplitude estimates from Stokes $Q, U$, and $V$. This assumes that all Stokes parameters experience the same scintillation amplitude variations, justified as there is no evidence for birefringence of scintillations (Simonetti et al. 1984). The average pulse profiles for the recent $10 \mathrm{~cm}$ observations of J1643-1224 are displayed in panel (D) of Figure 1. The residual of these profiles relative to a model formed from all the 2015 pre-March observations is displayed in panel (E) of Figure 1. Prior to 2015 March, the on-pulse residuals (phase $\phi=-0.2$ to $\phi \approx 0$ ) show nearly noise-like variations. Significant excess signal is observed on the leading edge of the pulse profile after 2015 March, commensal with the sudden change in residuals plotted in the bottom panel of the figure. The pulse profiles from the epochs immediately before (panel (F)) and after (panel (G)) highlight the emergence of the new component in the pulse profile. Neither the residual TOAs nor the residual profiles return to the pre-event levels, with $10 \mathrm{~cm}$ and $20 \mathrm{~cm}$ profiles showing permanent excess power on the leading edge of the profile, resulting in the post-event TOAs showing an apparent permanent offset of $-5 \mu \mathrm{s}$.

\section{PROFILE-DOMAIN TIMING}

We analyzed our observations using a recently developed profile-domain timing methodology that implements pulsartiming analyses directly on pulse profiles (Lentati et al. 2015; Lentati \& Shannon 2015). The algorithm incorporates a likelihood function that simultaneously describes deterministic and stochastic contributions to both the pulse profile and the TOAs. It uses Bayesian methodology to sample the posterior distribution, enabling robust parameter estimation and marginalization over other nuisance parameters. It also enables the quantitative comparison of families of models through the use of evidence (the integral of the likelihood weighted by the prior). When evidences are compared, differences in the log evidence of $\geqslant 3$ delineate a clear model preference with a probability of $\gtrsim 95 \%$. For differences in the log evidence of $\Delta \log E<3$, the simpler model is preferred. The method naturally accounts for the covariance between shape and timing variations and can therefore better break the degeneracy between the two. The models considered are listed in Table 1.

Nonstationary profile variations were parameterized using a shapelet basis (Refregier 2003), comprising a series of Hermite 
Table 1

Models and Evidences

\begin{tabular}{|c|c|c|c|c|c|c|c|}
\hline \multirow[b]{3}{*}{ Model } & \multicolumn{6}{|c|}{ Model } & \multirow[b]{3}{*}{$\Delta \log E$} \\
\hline & \multicolumn{2}{|c|}{ Shape Variations } & \multicolumn{4}{|c|}{ Timing Noise } & \\
\hline & Trans. (\#) & Perm. (\#) & Stat. & NS & Trans. & Perm. & \\
\hline 1 & 0 & 0 & $\checkmark$ & $x$ & $\checkmark$ & $\checkmark$ & -84.2 \\
\hline 2 & 0 & 0 & $\checkmark$ & $\checkmark$ & $\checkmark$ & $\checkmark$ & -88.0 \\
\hline 3 & 0 & 2 & $\checkmark$ & $\times$ & $\checkmark$ & $\checkmark$ & -33.4 \\
\hline 4 & $2 \mathrm{~g}$ & 0 & $\checkmark$ & $\times$ & $\checkmark$ & $\checkmark$ & -25.4 \\
\hline 5 & $2 \mathrm{e}$ & 0 & $\checkmark$ & $x$ & $\checkmark$ & $x$ & -9.7 \\
\hline 6 & $2 e$ & $\mathbf{0}$ & $\checkmark$ & $x$ & $\checkmark$ & $\checkmark$ & -2.0 \\
\hline 7 & $2 e$ & 2 & $\checkmark$ & $x$ & $x$ & $x$ & -2.7 \\
\hline 8 & $2 e$ & 2 & $\checkmark$ & $x$ & $\checkmark$ & $\checkmark$ & -1.3 \\
\hline 9 & $2 e$ & 2 & $\checkmark$ & $x$ & $x$ & $\checkmark$ & $\mathbf{0}$ \\
\hline 10 & $2 e$ & 2 & $\checkmark$ & $x$ & $\checkmark$ & $x$ & -2.5 \\
\hline 11 & $3 e$ & 2 & $\checkmark$ & $x$ & $\checkmark$ & $\checkmark$ & -4.3 \\
\hline 12 & $4 \mathrm{e}$ & 2 & $\checkmark$ & $x$ & $\checkmark$ & $\checkmark$ & -1.8 \\
\hline
\end{tabular}

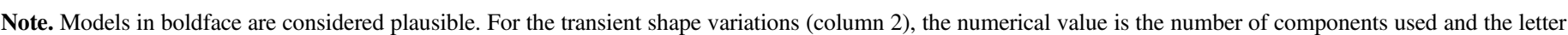

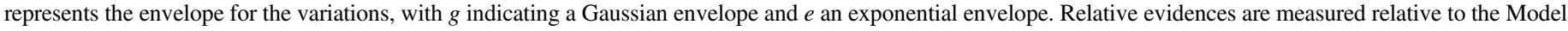
9, which has the highest evidence.

polynomials multiplied by a Gaussian. This basis provides a series of functions that compactly model pulse profiles and profile variations, using only two nonlinear parameters (the center of the profile variation and its width) for the entire basis.

We considered both permanent and transient changes to the pulse profile. For the transient changes, we compared models where the amplitude of the additional components were assumed to exponentially decay after an initial epoch, or to rise and fall like a Gaussian. These models are listed in the second (transient) and third (permanent) columns of Table 1.

In conjunction with the profile modeling, we also searched for timing noise, considering both stationary timing noise modeled using a power-law process and predicted to be present in many MSPs (Shannon \& Cordes 2010; fourth column of Table 1), and nonstationary timing noise which could be described using the shapelet basis (fifth column; see also Lentati et al. 2016).

Motivated by the shape of the perturbation as seen in the TOAs, we also considered TOA perturbations that, after an initial epoch, either exponentially decayed (sixth column of Table 1), similar to the glitch decays observed in many pulsars (Yu et al. 2013), or resulted in a permanent offset (seventh column). A permanent offset would be indicative of an unmodeled transient change in the spin state. When the same type of TOA perturbations and shape variations (i.e., permanent or exponential) were modeled simultaneously, the onset epoch and decay timescales were modeled as being common to both. While changes in shape and TOA may not necessarily share the same physical timescale, we used the common model parameters to ensure preference for either shifts or shape changes could be determined from the posterior parameter estimates.

In order to reduce computation requirements, only the most recent 4 years of observation (see Figure 1) were considered, we analyzed the $10 \mathrm{~cm}$ and $20 \mathrm{~cm}$ data sets independently, and we did not consider the $50 \mathrm{~cm}$ observations. We found that the data strongly support a transient change in the pulse profile, with exponential models (Models 5-12 in Table 1) favored over those with Gaussian variation (Model 4). The shape variations can be adequately modeled using only two shapelet components, with the evidence decreasing as additional components are added to the model.

A nonstationary timing noise origin for the TOA variations (Model 2) is strongly disfavored. In addition, we find that the data support a model where no TOA shifts are required in addition to the shape variation (Model 7).

Our Stokes-I observations cannot distinguish between a permanent change in the pulse profile or a permanent offset in the TOAs (Models 6 and 7); however, the lack of an offset in the arrival times of the other Stokes components suggest that the profile has changed shape. In this case, we limit the shift in arrival times to be $<1 \mu \mathrm{s}$. After accounting for the pulse-profile variations (and possible permanent offset) we find no evidence for excess noise in the TOAs. Evidence comparisons of models in the $20 \mathrm{~cm}$ band result in the same conclusions.

In Figure 3, we show the posterior distribution for the shape variations in both the $10 \mathrm{~cm}$ and $20 \mathrm{~cm}$ bands. We find that the components decay with a common timescale $\tau$, but the $20 \mathrm{~cm}$ component is broader than that observed at $10 \mathrm{~cm}$.

After accounting for the shape variations results, the TOAs are less biased, extrapolating from the long-term model. In panel (C) of Figure 1, we show the perturbations associated with one of the favored pulse-shape variation models (Model 7) as a red line and a maximum-likelihood representation of the corrected TOAs. The blue points show the residuals corrected by the model and do not show any variation or permanent offset.

\section{DISCUSSION AND CONCLUSIONS}

Secular shape variations have not hitherto been observed in an MSP. In state-changing young pulsars, pulse shapes change between discrete modes (Lyne et al. 2010), with strong correlations identified between change in pulse shape and rotation state. The shape variations observed in PSR J1643 -1224 are most reminiscent of those observed in the young pulsar B0736-40 (Karastergiou et al. 2011), in which a new component suddenly appeared and gradually drifted toward the central emission region over a few years of observation. In both PSRs J1643-1224 and B0736-40, the new components are unpolarized. The lack of a change in spin down (manifested as 


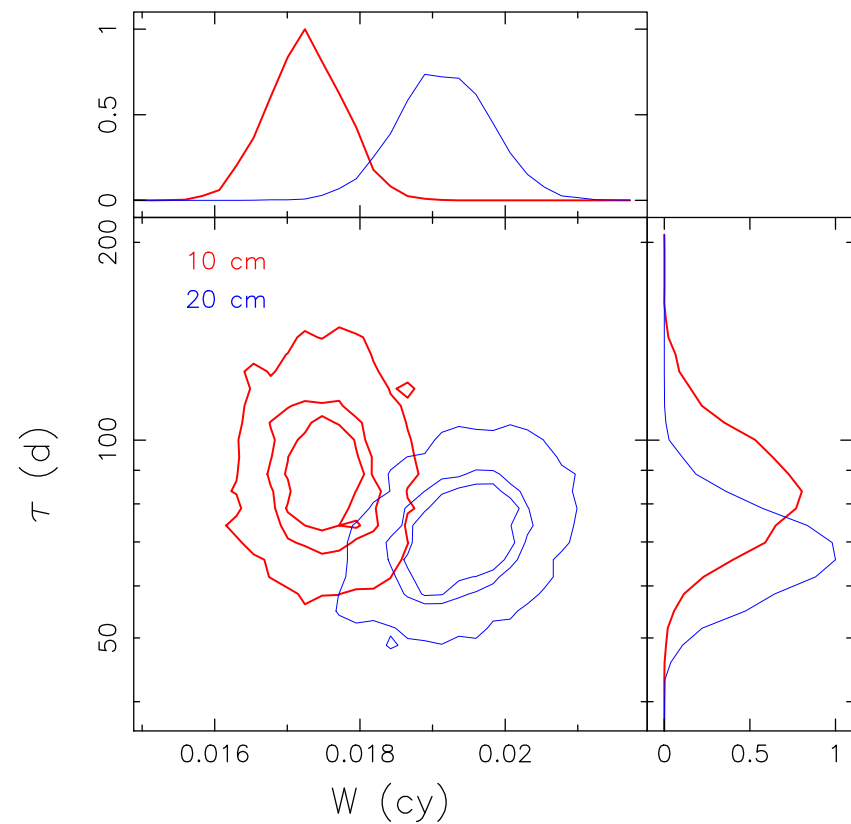

Figure 3. Marginalized posterior distributions for decay time $(\tau)$ and width $(W)$, as measured via profile-domain timing of our $10 \mathrm{~cm}$ (red) and $20 \mathrm{~cm}$ (blue) data.

an offset in the TOAs) is in contrast to what is observed in other pulsars like PSR B0736-40. A mean change in the spindown rate of $\langle\Delta \dot{\nu}\rangle$ over a timescale $T$ would result in a permanent offset in pulse phase $\Delta \Phi \approx\langle\Delta \dot{\nu}\rangle T^{2} / 2$. Because the shift in the arrival times is limited to $<1 \mu$ s after 100 days, the change in spindown is $|\Delta \dot{\nu}| \lesssim 2 \times 10^{-20} \mathrm{~s}^{-2}$, which is a factor of $10^{4}$ smaller than the long-term spindown of the pulsar (Reardon et al. 2016) and two orders of magnitude fractionally smaller than the correlated spindown variations observed in other pulsars.

In PSR B0736-40, the profile variation was attributed to the impact of an asteroid on the neutron-star magnetosphere (Brook et al. 2014). It is unlikely that an extrinsic interaction is the cause of the profile variation observed here. The magnetospheres of MPSs are factors of 10-100 smaller than that of slower-rotating pulsars like B0736-40, making asteroidal intrusion a less likely explanation for the profile variation (Cordes \& Shannon 2008). Furthermore, compared to solitary pulsars, binary systems such as PSR J1643-1224 potentially harbor less massive and wider disks (Shannon et al. 2013) with fewer mechanisms to dynamically inject material into the pulsar magnetosphere.

For gravitational-wave detection experiments, it is essential to search for profile variability and correct for it, if it exists. As an example, we placed a limit on the gravitational-wave background in the four most recent years of $10 \mathrm{~cm}$ PDFB4 observations of PSR J1643-1224 using both standard methods and profile-domain timing. Limits placed in the profile domain follow methods presented in Lentati \& Shannon (2015) and limits in the TOA domain follow methods presented in Shannon et al. (2015). We assumed a background with a strain spectrum of the form $h_{\mathrm{c}}(f)=A_{\mathrm{c}, \mathrm{yr}} f^{-2 / 3}$, where $f$ is the frequency measured in cycles per year and $A_{\mathrm{c}, \mathrm{yr}}$ is the amplitude of the background. This is the form predicted for a background produced by binary supermassive black holes that are radiating all of their energy in gravitational radiation. When neglecting to account for the profile variations, we find $A_{\mathrm{c}}$, yr $<2 \times 10^{-12}$. When accounting for the variability, the limit improved to $A_{\mathrm{c}, \mathrm{yr}}<9 \times 10^{-14}$. Improvements to other pulsars will depend on the properties and signal-to-noise ratio of the profile variations and the spectral color that the variations induce in the TOAs.

It is possible that shape variations induce the timing noise observed in many MSPs. Red timing noise has been identified as being common in MSPs (Manchester et al. 2013; Arzoumanian et al. 2015; Caballero et al. 2016; Reardon et al. 2016). In contrast to observations of timing noise in young pulsars and prediction for MSPs (and in observed in a minority; Shannon \& Cordes 2010), much of the observed noise in MSPs has a relatively shallow or non-power-law spectral shape (Arzoumanian et al. 2015; Reardon et al. 2016). If this noise is associated with shape variations, it may be possible to mitigate it. The ability to correct for shape variations will depend on the signal-to-noise ratio of the variations. High-fidelity observations from large-aperture telescopes like the Five-hundredmetre aperture spherical telescope (Nan et al. 2011; Hobbs et al. 2014) and the Square Kilometre Array (Janssen et al. 2015) will be particularly amenable to the identification and correction of these magnetospheric disturbances.

The Parkes radio telescope is part of the Australia Telescope, which is funded by the Commonwealth of Australia for operation as a National Facility managed by the Commonwealth Science and Industrial Research Organization (CSIRO). The Parkes Pulsar Timing Array Project project was initiated with support from an Australian Research Council (ARC) Federation Fellowship (FF0348478) to R.N.M. and from the CSIRO under that fellowship program. The PPTA project has also received support from the ARC through Discovery Project grants DP0985272 and DP140102578. N.D.R.B. acknowledge support form a Curtin University research fellowship. G.H. and Y.L. are recipients of ARC Future Fellowships (respectively, FT120100595 and FT110100384). S.O. is supported by the Alexander von Humboldt Foundation. R.M.S. acknowledges travel support from the CSIRO through a John Philip award for excellence in early career research.

\section{REFERENCES}

Arzoumanian, Z., Brazier, A., Burke-Spolaor, S., et al. 2015, ApJ, 813, 65 Brook, P. R., Karastergiou, A., Buchner, S., et al. 2014, ApJL, 780, L31 Caballero, R. N., Lee, K. J., Lentati, L., et al. 2016, MNRAS, 457, 4421 Cordes, J. M., \& Shannon, R. M. 2008, ApJ, 682, 1152

Cordes, J. M., \& Shannon, R. M. 2010, arXiv:1010.3785

Dai, S., Hobbs, G., Manchester, R. N., et al. 2015, MNRAS, 449, 3223

Demorest, P. B., Pennucci, T., Ransom, S. M., Roberts, M. S. E., \& Hessels, J. W. T. 2010, Natur, 467, 1081

Edwards, R. T., Hobbs, G. B., \& Manchester, R. N. 2006, MNRAS, 372, 1549 Foster, R. S., \& Backer, D. C. 1990, ApJ, 361, 300

Hobbs, G., Archibald, A., Arzoumanian, Z., et al. 2010, CQGra, 27, 084013 Hobbs, G., Dai, S., Manchester, R. N., et al. 2014, arXiv:1407.0435

Hotan, A. W., Bailes, M., \& Ord, S. M. 2004a, MNRAS, 355, 941

Hotan, A. W., van Straten, W., \& Manchester, R. N. 2004b, PASA, 21, 302 Janssen, G., Hobbs, G., McLaughlin, M., et al. 2015, in Advancing Astrophysics with the Square Kilometre Array (AASKA14) (Giardini Naxos, Italy), 37

Karastergiou, A., Roberts, S. J., Johnston, S., et al. 2011, MNRAS, 415, 251 Kramer, M., Stairs, I. H., Manchester, R. N., et al. 2006, Sci, 314, 97 Kramer, M., Xilouris, K. M., Camilo, F., et al. 1999, ApJ, 520, 324 Lentati, L., Alexander, P., \& Hobson, M. P. 2015, MNRAS, 447, 2159 Lentati, L., Alexander, P., Hobson, M. P., et al. 2014, MNRAS, 437, 3004 Lentati, L., \& Shannon, R. M. 2015, MNRAS, 454, 1058

Lentati, L., Shannon, R. M., Coles, W. A., et al. 2016, MNRAS, 458, 2161 
Liu, K., Karuppusamy, R., Lee, K. J., et al. 2015, MNRAS, 449, 1158

Lyne, A., Hobbs, G., Kramer, M., Stairs, I., \& Stappers, B. 2010, Sci, 329,408

Manchester, R. N., Hobbs, G., Bailes, M., et al. 2013, PASA, 30, 17

Nan, R., Li, D., Jin, C., et al. 2011, IJMPD, 20, 989

Ramachandran, R., \& Kramer, M. 2003, A\&A, 407, 1085

Reardon, D. J., Hobbs, G., Coles, W., et al. 2016, MNRAS, 455, 1751

Refregier, A. 2003, MNRAS, 338, 35

Rickett, B. J. 1990, ARA\&A, 28, 561
Shannon, R. M., \& Cordes, J. M. 2010, ApJ, 725, 1607

Shannon, R. M., Cordes, J. M., Metcalfe, T. S., et al. 2013, ApJ, 766, 5

Shannon, R. M., Ravi, V., Lentati, L. T., et al. 2015, Sci, 349, 1522

Shao, L., Caballero, R. N., Kramer, M., et al. 2013, CQGra, 30, 165019

Simonetti, J. H., Cordes, J. M., \& Spangler, S. R. 1984, ApJ, 284, 126

Tiburzi, C., Hobbs, G., Kerr, M., et al. 2016, MNRAS, 455, 4339

van Straten, W. 2013, ApJS, 204, 13

Weisberg, J. M., Romani, R. W., \& Taylor, J. H. 1989, ApJ, 347, 1030

Yu, M., Manchester, R. N., Hobbs, G., et al. 2013, MNRAS, 429, 688 